\title{
Differences in tumor microenvironments between primary lung tumors and brain metastases in lung cancer patients: therapeutic implications for immune checkpoint inhibitors
}

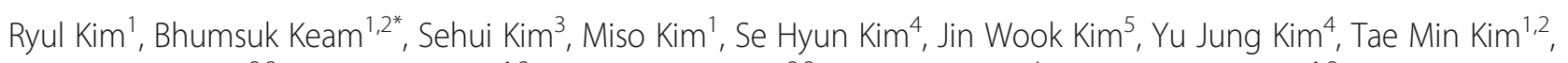
Yoon Kyung Jeon ${ }^{2,3}$, Dong-Wan Kim ${ }^{1,2}$, Doo Hyun Chung ${ }^{2,3}$, Jong Seok Lee ${ }^{4}$ and Dae Seog Heo ${ }^{1,2}$

\begin{abstract}
Background: We aimed to compare intra- and extracranial responses to immune checkpoint inhibitors (ICIs) in lung cancer with brain metastases (BM), and to explore tumor microenvironments of the brain and lungs focusing on the programmed cell death-1 (PD-1)/programmed cell death ligand-1 (PD-L1) pathway.

Methods: Two cohorts of lung cancer patients with BM were analyzed. Cohort 1 included 18 patients treated with nivolumab or pembrolizumab, and intra- and extracranial responses were assessed. Cohort 2 comprised 20 patients who underwent both primary lung surgery and brain metastasectomy. Specimens from cohort 2 were subjected to immunohistochemical analysis for the following markers: CD3, CD4, CD8, FOXP3, and PD-1 on tumor infiltrating lymphocytes (TIL) and PD-L1 on tumor cells.
\end{abstract}

Results: Seven patients (38.9\%) in cohort 1 showed progressive disease in both primary and intracranial lesions. Although the other 11 patients exhibited a partial response or stable disease in the primary lesion, eight showed a progression in $\mathrm{BM}$. Interestingly, $\mathrm{PD}-1^{+}$TILs were significantly decreased in $\mathrm{BM}(P=0.034)$. For fifteen patients with adenocarcinoma, more distinctive patterns were observed in $\mathrm{CD}^{+}(P=0.078), \mathrm{CD}^{+}(P=0.055), \mathrm{FOXP3}^{+}(P=0.016)$, and PD- $1^{+}(P=0.016)$ TILS.

Conclusions: There may be discordant responses to an $\mathrm{ICl}$ of lung cancer between primary lung lesion and BM based on discrepancies in the tumor microenvironment. The diminished infiltration of PD-1 ${ }^{+}$TILs in tumor tissue within the brain may be one of the major factors that hinder the response to anti-PD-1 antibody in BM.

Keywords: Immunotherapy, Lung cancer, Brain metastasis, PD-1, PD-L1

\section{Background}

About 30\% of advanced lung cancer patients develop brain metastases (BM), and their prognosis as well as quality of life are generally poor $[1,2]$. The standard management for these patients includes metastasectomy and radiotherapy-

\footnotetext{
* Correspondence: bhumsuk@snu.ac.kr

'Department of Internal Medicine, Seoul National University Hospital, 101 Daehak-ro, Jongno-gu, Seoul 03080, Republic of Korea

${ }^{2}$ Cancer Research Institute, Seoul National University College of Medicine,

101 Daehak-ro, Jongno-gu, Seoul 03080, South Korea

Full list of author information is available at the end of the article
}

either stereotactic radiosurgery or whole brain radiotherapy (WBRT). However, despite an improved local control rate [3], such localized treatments have inevitable toxic effects, including cognitive decline or a deterioration in the patient's quality of life [4-6]. Furthermore, local treatment can delay the initiation of systemic treatment, which is crucial in patients with rapidly progressive disease $[7,8]$. Accordingly, systemic treatment is recommended for patients with $\mathrm{BM}$ who are asymptomatic or experience only minimal neurological symptoms; this may provide a benefit for BM while simultaneously treating extracranial disease [9].

(c) The Author(s). 2019 Open Access This article is distributed under the terms of the Creative Commons Attribution 4.0 International License (http://creativecommons.org/licenses/by/4.0/), which permits unrestricted use, distribution, and 
Recently, immune checkpoint inhibitors (ICIs) have emerged as a promising new treatment in various cancer types. These drugs, including anti-cytotoxic T-lymphocyte antigen 4 (CTLA-4) antibodies and anti-programmed cell death-1 (anti-PD-1)/programmed cell death ligand-1 (PD-L1) antibodies, reactivate the anti-tumor immunity of $\mathrm{T}$ cells $[10,11]$. Although the efficacy as a first-line treatment in metastatic non-small-cell lung cancer (NSCLC) was not evident yet, [12] nivolumab have been approved by the US Food and Drug Administration (FDA) for the treatment of metastatic NSCLC and has become standard treatment in a second-line setting. [13-17]. However, most of these trials have excluded patients with any history of BM or active/ symptomatic BM requiring steroid treatment. Furthermore, the evidence for immune checkpoint inhibitors in the management of BM has been largely limited to retrospective analyses of melanoma metastases and ipilimumab [18]. Therefore, the intracranial efficacy of ICIs is relatively unknown and has not yet been validated.

In this regard, prospective clinical trials of ICIs are currently under way, and tentative results suggested activity [18]. Recent early phase clinical trials have conducted to investigate the therapeutic efficacy of ICIs on BM in patients with NSCLC or melanoma $[19,20]$. Although they reported that pembrolizumab and ipilimumab can have therapeutic activity on BM in patients with NSCLC and melanoma, many patients did not showed response in the brain or in extracranial sites. Further more, because these studies mainly included patients without neurological symptoms or the need for corticosteroids, the efficacy of ICIs on BM in real world are still inconclusive yet. Additional studies addressing combination treatment strategies and biomarker development are necessarily warranted.

Over the past decade, the tumor microenvironment (TME) has emerged as a critical regulator determining the response of ICIs [21]. Because of the distinctive composition of the extracellular matrix (ECM) as well as immunological environments in the brain, the TME of the brain has unique features that distinguishes it from the TME in primary lung cancer [22]. For example, unusual tissue-resident cell types, including microglia, astrocytes and neurons, are present in the brain's TME [23], Furthermore, more importantly, the brain is one of the immune privileged organs that must be sheltered from immune cell entry and/or attack. Therefore, the TME of an early-stage brain tumor is generally immunosuppressive with essentially no trafficking or patrolling by peripheral immune cells [23]. Because immuno-oncology strategies rely on the sophisticated interaction between the tumor and its microenvironment, such a peculiar immunological environment of the brain presents a formidable challenge to overcome.
In the era of immunotherapy, understanding how BM are influenced by the immunological peculiarities of the brain will be crucial to forging therapeutic advances in lung cancer. The purposes of this study are 1) to compare intracranial and extracranial responses to ICIs in lung cancer patients with BM, and 2) to investigate differences in the TME between lung and brain metastases, focusing on the PD-1/PD-L1 pathway.

\section{Methods \\ Patients and samples}

Two independent cohorts of lung cancer patients were retrospectively analyzed to investigate the differences between primary tumors and BM in two respects: cohort 1 for comparing extra/intracranial responses to anti-PD-1 antibody; cohort 2 for comparing differences in the immunological TME. Cohort 1 included advanced lung cancer patients who were treated with intravenous nivolumab or pembrolizumab as part of an expanded access program (EAP), Keynote 010 trial (NCT01905657), or in routine practice from February 2014 to November 2016 at Seoul National University Hospital (SNUH) and Seoul National University Bundang Hospital. Treatment continued until disease progression, unacceptable toxicity that precluded continuing drug treatment, or death. Patients were allowed to continue treatment despite disease progression if they were deriving a clinical benefit according to an investigator's assessment. Patients were eligible for treatment if they had at least one $\mathrm{BM}$, with the longest diameter being $\geq 5$ $\mathrm{mm}$, before or during treatment with anti-PD-1 antibody. Patients who received local treatment for previously known BM were not excluded. Cohort 2 included lung cancer patients who underwent both brain metastasectomies for their BM, and primary lung surgery. Therefore, patients who were available for paired lung cancer and BM surgical specimens were enrolled. The Tissue Registry at SNUH was searched between June 2011 and November 2016. Two expert pathologists (S.H.K. and Y.K.J) reviewed tissue sections for adequacy. This study was approved by the SNUH Institutional Review Board (IRB approval number: $\mathrm{H}-1702-158-836)$ and was conducted in accordance with Declaration of Helsinki provisions.

\section{Response evaluation}

A systemic response to anti-PD-1 antibody was measured by standard Response Evaluation Criteria in Solid Tumors (RECIST; version 1.1). An intracranial response was assessed by brain gadolinium-enhanced magnetic resonance imaging (MRI), using RECIST modified to allow target central nerve system lesions, $5 \mathrm{~mm}$ or larger in maximum diameter, and with up to five BMs permitted (modified RECIST) [24]. Representative MRI images for response evaluations are available in Additional file 1: Figure S1. 


\section{Immunohistochemistry}

Formalin-fixed paraffin-embedded specimens were examined by immuno-staining tumor cells and TILs. Immunohistochemistry (IHC) was performed using the following antibodies: rabbit anti-PD-L1 (E1L3N) XP $\mathrm{XAb}^{\oplus}$ (Cell Signaling Technology, Danvers, MA, USA), mouse anti-CD3 mAb (DAKO, Santa Clara, CA, USA), mouse anti-CD4 mAb (Cell Marque, Rocklin, CA, USA), rabbit anti-CD8 mAb (Thermo Scientific Fischer, Rockford, IL, USA), mouse anti-PD-1 mAb (Cell Marque, Rocklin, CA, USA) and mouse anti-FOXP3 mAb (Abcam, Cambridge, UK). IHC for TILs, except for $\mathrm{CD}^{+}$TILs, was performed using a Benchmark XT autostainer (Ventana Medical Systems, Basel, Switzerland). In the case of $\mathrm{CD}^{+} \mathrm{IHC}$, a Bond-Max automated immunostainer (Leica Microsystems, Melbourne, Australia) was used. PD-L1 IHC was evaluated based on the intensity and proportion of membranous staining, with or without cytoplasmic staining, in tumor cells and was scored as follows: $0+$ (no appreciable staining above background), 1+ (weak membranous staining and/or cytoplasmic staining), 2+ (moderate membranous staining and/or cytoplasmic staining), and 3+ (strong membranous staining and/or cytoplasmic staining) [25]. Each score was multiplied by the percentage of cells (0$100 \%)$ to achieve a $\mathrm{H}$-score; $\mathrm{H}$-score $=(\%$ of cells $3+) \times 3+$ (\% of cells $2+) \times 2+(\%$ of cells $1+$ ). All slides were blinded with respect to clinical characteristics and outcomes, and were reviewed and scored by two experienced pathologists (S.H.K. and, Y.K.J.).

\section{Automated enumeration of tumor-infiltrating lymphocytes}

To determine the amount of $\mathrm{CD}^{+}, \mathrm{CD}^{+}, \mathrm{CD}^{+}, \mathrm{PD}-1^{+}$ and $\mathrm{FOXP3}^{+}$TILs, representative slides were immunostained for CD3, CD4, CD8, PD-1 and FOXP3 and scanned for virtual microscopy using an Aperio ScanScope (Aperio Technologies, Vista, CA, USA). The number of TILs was automatically counted in intact tumor areas lacking necrosis using modified nuclear IHC algorithms in Aperio ImageScope software, as previously described [25]. The mean value of TILs per unit area $\left(\mathrm{mm}^{2}\right)$ was calculated.

\section{Statistical analysis}

Extra- and intracranial responses to anti-PD-1 antibody were descriptively analyzed. Differences in IHC markers between primary and BM were assessed using the paired Wilcoxon rank-sum test. Correlations between IHC markers were evaluated by Spearman's rank correlation analysis. Survival analyses were performed for cohort 2 using the Kaplan-Meier method and were compared using a log-rank test. Overall survival (OS) was measured from the date of brain metastasectomy until either death due to any cause or the last follow-up date. A Cox proportional hazard regression model was applied to determine the hazard ratio (HR) for specific variables with respect to OS. For all statistical analyses, two-sided $P$-values $<0.05$ were considered statistically significant. All statistical analyses were carried out using $\mathrm{R}$ version 3.3.2 (http://www.r-project.org).

\section{Results \\ Demographics of study subjects}

The clinicodemographic characteristics of patients in the two study cohorts are summarized in Table 1 . Cohort 1 included 18 lung cancer patients who had BM before or during anti-PD-1 treatment. Most patients had an adenocarcinoma histology (66.7\%), and stages IIIA-IV disease $(83.3 \%)$. Three patients $(16.7 \%)$ received cerebral irradiation by WBRT during treatment with an ICI. Cohort 2 included 20 lung cancer patients who underwent brain metastasectomy, and therefore paired primary and BM specimens were available. Seven pairs (35.0\%) were obtained from cases with synchronous disease at their initial diagnosis, and 13 pairs $(65.0 \%)$ were obtained from patients with metachronous disease who underwent metastasectomy 5 or more months after the initial diagnosis. The median interval between acquisitions of paired lesions was 17 months (range 0-81 months). No patient had been exposed to anti-PD-1 antibody in cohort 2 , and five patients $(25.0 \%)$ underwent cerebral irradiation before the acquisition of specimens.

Analysis of cohort 1: Comparison of response to anti-PD1 antibody between primary lung cancer and brain metastasis

Of 18 patients in cohort 1, the primary lung lesion had progressed in seven patients (38.9\%). All such patients showed progressive disease in intracranial lesions (Fig. 1). The primary lesion showed a stable disease $(n=9$, $50.0 \%)$ and partial response $(n=2,11.1 \%)$ in 11 patients, with eight of these exhibiting progressive disease in BM. Although two patients showed a partial response in brain tumors, they were found to have received WBRT with nivolumab. Collectively, these results implied that $\mathrm{BM}$ was poorly responsive to anti-PD-1 antibody compared with the primary lung lesion.

\section{Analysis of cohort 2: Comparison of immunological tumor microenvironment between primary lung cancer and brain metastasis}

On the hypothesis that the distinctive immunological TME of the brain determines the response to the anti-PD-1 antibody, the amounts of TILs and PD-L1 expression on tumor cells in the brain TME were compared with those of primary lung cancer specimens (Fig. 2). Only twelve (60.0\%) out of 20 patients in cohort 2 were eligible for a paired comparison because lung biopsy specimens obtained from the other 
Table 1 Clinicodemographic characteristics of patients in cohorts 1 and 2. Cohort 1 consisted of 18 lung cancer patients who underwent brain metastasectomy for metastatic brain tumors, while cohort 2 included 20 lung cancer patients with brain metastases who were treated with anti-PD-1 antibody (nivolumab or pembrolizumab). The two patient cohorts were independent of each other

\begin{tabular}{|c|c|c|}
\hline Characteristics & Cohort $1(N=18)$ & Cohort $2(N=20)$ \\
\hline Age, median (range) & $59(32-76)$ & $60(33-77)$ \\
\hline \multicolumn{3}{|l|}{ Sex, n (\%) } \\
\hline Male & $12(66.7)$ & $10(50)$ \\
\hline Female & $6(33.3)$ & $10(50)$ \\
\hline \multicolumn{3}{|l|}{ Smoking, n (\%) } \\
\hline Current smoker & $4(22.2)$ & $7(35.0)$ \\
\hline Ex-smoker & $6(33.3)$ & $3(15.0)$ \\
\hline Never smoked & $8(44.4)$ & $10(50.0)$ \\
\hline \multicolumn{3}{|l|}{ Stage at diagnosis ${ }^{\mathrm{a})}, \mathrm{n}(\%)$} \\
\hline$|A-| \mid B$ & $1(5.6)$ & $4(20.0)$ \\
\hline IIIA-IV & $15(83.3)$ & $16(80.0)$ \\
\hline Extensive disease (for small cell lung cancer) & $2(11.1)$ & $0(0.0)$ \\
\hline \multicolumn{3}{|l|}{ Histology, n (\%) } \\
\hline Adenocarcinoma & $12(66.7)$ & $15(75.0)$ \\
\hline Squamous cell carcinoma & $1(5.6)$ & $4(20.0)$ \\
\hline Sarcomatoid carcinoma & $2(11.1)$ & $1(5.0)$ \\
\hline Small cell lung cancer & $2(11.1)$ & $0(0.0)$ \\
\hline \multicolumn{3}{|l|}{ Mutational status, n (\%) } \\
\hline EGFR & $3(16.7)$ & $9(45.0)$ \\
\hline ALK & $2(11.1)$ & $0(0.0)$ \\
\hline \multicolumn{3}{|l|}{ Temporal relationship, n (\%) } \\
\hline Synchronous & NA & $7(35.0)$ \\
\hline Metachronous & NA & $13(65.0)$ \\
\hline \multicolumn{3}{|l|}{ Anti-PD-1 antibody treatment } \\
\hline Nivolumab & $14(77.8)$ & NA \\
\hline Pembrolizumab & $4(22.2)$ & NA \\
\hline \multicolumn{3}{|c|}{ No. of chemotherapy treatments before EAP or brain surgery, $\mathrm{n}(\%)$} \\
\hline 0 & $0(0.0)$ & $8(40.0)$ \\
\hline 1 & $6(33.3)$ & $7(35.0)$ \\
\hline 2 & $7(38.9)$ & $2(10.0)$ \\
\hline$\geq 3$ & $5(27.8)$ & $3(15.0)$ \\
\hline \multicolumn{3}{|c|}{ Cerebral irradiation before EAP or brain surgery, n (\%) } \\
\hline Whole brain radiotherapy & $3(16.7)$ & $2(10.0)$ \\
\hline Radiosurgery & $4(22.2)$ & $2(10.0)$ \\
\hline Both & $3(16.7)$ & $1(5.0)$ \\
\hline \multicolumn{3}{|l|}{ Cerebral irradiation during EAP, n (\%) } \\
\hline Whole brain radiotherapy & $3(16.7)$ & NA \\
\hline Radiosurgery & $0(0.0)$ & NA \\
\hline Both & $0(0.0)$ & NA \\
\hline
\end{tabular}

TTM classification according to the 7th edition of the AJCC cancer staging manual

Abbreviations: EGFR Epidermal growth factor receptor, $A L K$ anaplastic lymphoma kinase, $P D-1$ programmed cell death-1, AJCC American Joint Committee on Cancer, EAP expanded access program 

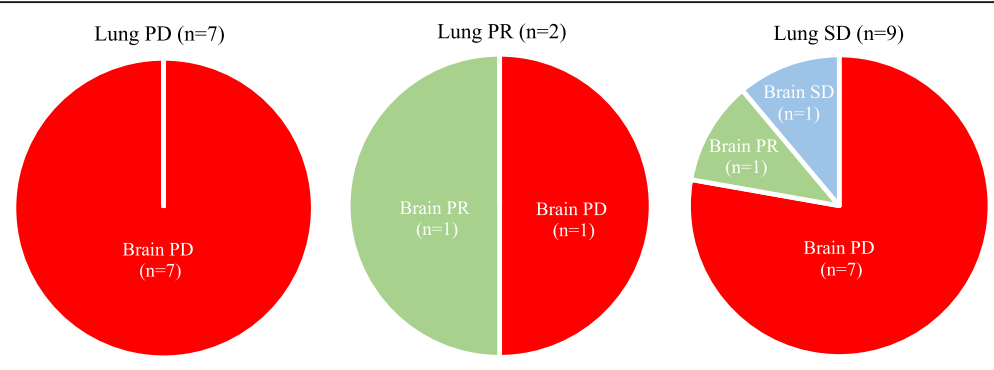

Patient ID
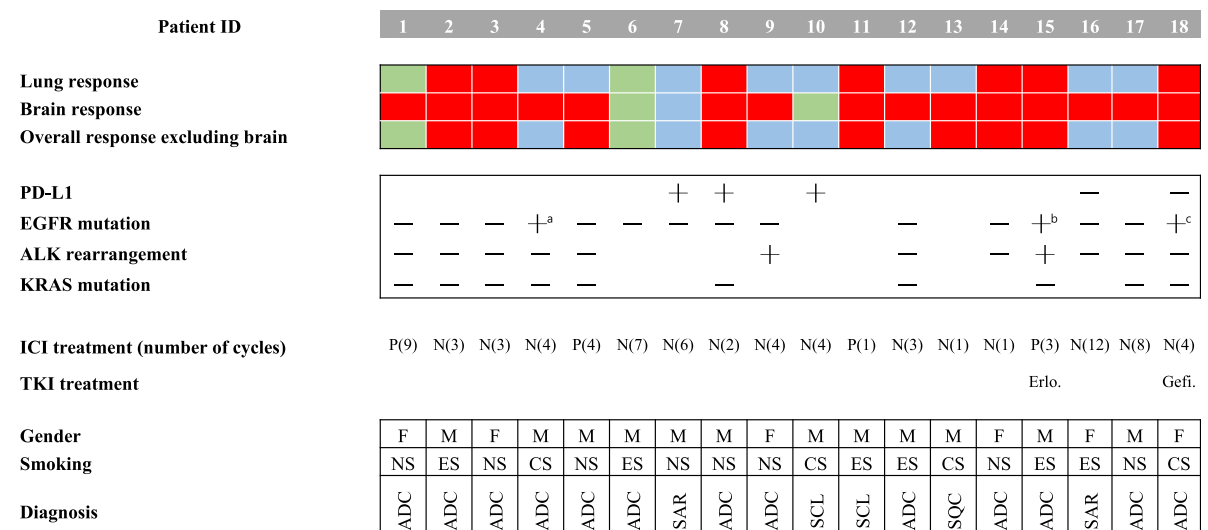

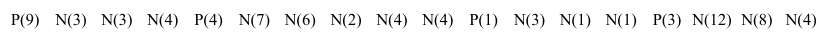
Erlo. Gefi.

\begin{tabular}{|c|c|c|c|c|c|c|c|c|c|c|c|c|c|c|c|c|c|}
\hline $\mathrm{F}$ & M & $\mathrm{F}$ & $\mathrm{M}$ & $\mathrm{M}$ & M & $\mathrm{M}$ & M & $F$ & $\mathrm{M}$ & $\mathrm{M}$ & $\mathrm{M}$ & $\mathrm{M}$ & $\mathrm{F}$ & $\mathrm{M}$ & $\mathrm{F}$ & M & $\mathrm{F}$ \\
\hline NS & ES & NS & CS & NS & ES & NS & NS & NS & CS & ES & ES & CS & NS & ES & ES & NS & $\mathrm{CS}$ \\
\hline 虽 & 虽 & 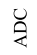 & 这 & 过 & 荠 & 丞 & $\begin{array}{l}\text { } \\
\text { Z }\end{array}$ & 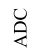 & $\vec{U}$ & $\vec{U}$ & 荤 & $\underset{d}{O}$ & 虽 & 过 & 丞 & 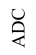 & $\begin{array}{l}\text { } \\
\text { \& }\end{array}$ \\
\hline
\end{tabular}

Fig. 1 Treatment response to anti-PD-1 antibody in cohort 1. (Upper) Pie charts demonstrating the response in primary lung cancer and paired brain metastases. (Lower) The treatment response along with clinicodemographic characteristics of individual patients in cohort 1 . Each patient received either nivolumab $(N)$ or pembrolizumab $(P)$ for the specified number of cycles. Each tile in this figure denotes the treatment response: red for progressive disease, blue for stable disease, and green for a partial response. Abbreviations: PD, progressive disease; PR, partial response; SD, stable disease; ID, identification number; P, pembrolizumab; N, nivolumab; PD-L1, programmed cell death ligand-1; PD-1, programmed cell death-1; M, male; F, female; EGFR, epidermal growth factor receptor; ALK, anaplastic lymphoma kinase; NS, never smoked; ES, ex-smoker; CS, current smoker; ADC, adenocarcinoma; SAR, pulmonary sarcomatoid carcinoma; SCL, small-cell lung cancer; SQC, squamous cell carcinoma, Erlo., erlotinib; Gefi., gefitinib; ICl, immune checkpoint inhibitor; TKl, tyrosine kinase inhibitor. a: Exon 20 insertion. b: Exon 19 deletion. c: L858R mutation

eight patients were too small to evaluate the TME; Three of them had synchronous BM, while the others had metachronous BM. The median interval between acquisitions of paired lesions in patients metachronous $\mathrm{BM}$ was 23.4 months (range $4.7-81.5$ months). The eight patients were not excluded from cohort 2 because they were eligible for survival analysis. The amounts of $\mathrm{CD}^{+}, \mathrm{CD}^{+}$, and $\mathrm{CD} 8^{+}$TILs, and the PD-L1 H-score of tumor cells did not show statistically significant differences between primary lung cancers and BM tissues, with $P$ values of $0.148,0.123,0.622$ and 0.675 , respectively. On the other hand, FOXP3 ${ }^{+}$TILs showed a decreasing tendency in the intracranial lesion $(P=0.077)$, and, more interestingly, PD- $1^{+}$TILs decreased in the brain in a statistically significant manner $(P=0.034)$. For fifteen patients with adenocarcinoma (75.0\%), more distinctive patterns were observed in $\mathrm{CD}^{+}(P=0.078)$, $\mathrm{CD}^{+}(P=0.055), \mathrm{FOXP}^{+}(P=0.016)$, and $\mathrm{PD}^{+}(P=$ 0.016) TILs (Additional file 2: Figure S2). Figure 3 shows representative images of a male patient (ID 17 of cohort 2) with adenocarcinoma histology. In the BM specimen of the patient, PD- $1^{+}$and $\mathrm{CD} 8^{+}$TILs were dramatically decreased, while PD-L1 expression on tumor cells was relatively unchanged. The infiltration of $\mathrm{CD}^{+}$TILs correlated positively with the PD-L1 $\mathrm{H}$-score of tumor cells in the primary lung cancer (Spearman $\rho=0.545, P=0.083$; Additional file 3: Figure $\mathrm{S} 3 \mathrm{~A}$ ), and BM (Spearman $\rho=0.444, P=0.049$; Additional file 3: Figure S3B). No correlation between PD1 + TILs and PD-L1 expression was noted in the primary lung cancer Spearman $\rho=0.116, P=0.720$; Additional file 3: Figure S3C). In contrast, the infiltration of PD1+ TILs positively correlated with PD-L1 expression of tumor cells in BM (Spearman $\rho=0.550, P=0.012$; Additional file 3: Figure S3D). For patients with metachronous disease, there was no significant correlation between PD- $1^{+}$TIL infiltration on brain TME and the interval of development of BM. (Spearman $\rho=0.133, \quad P=0.744$; Additional file 4: Figure S4). Altogether, these results revealed that the brain TME differed from that of the primary cancer in terms of an immunological environment, with a markedly decreased amount of PD- $1^{+}$TILs. The 

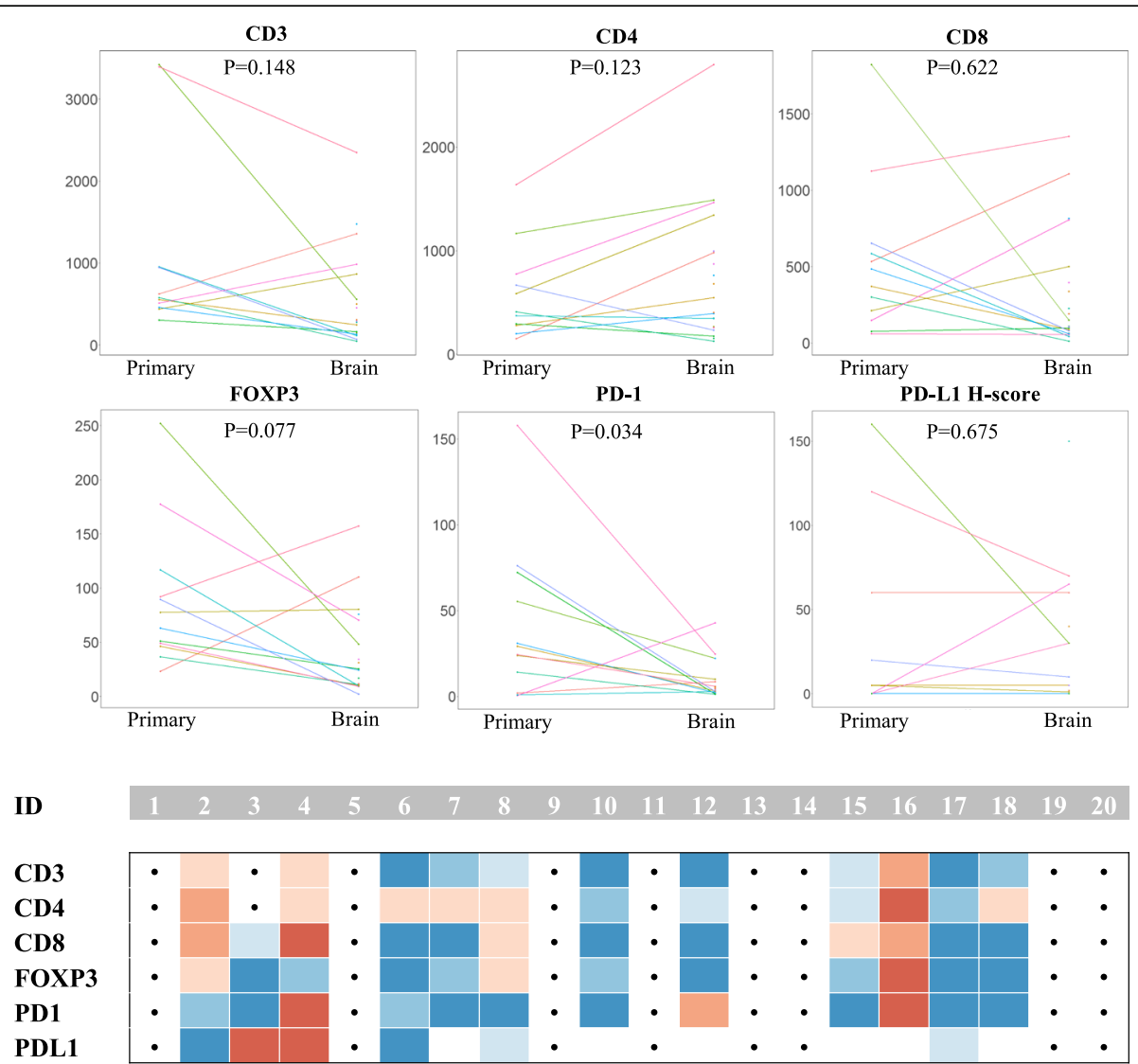

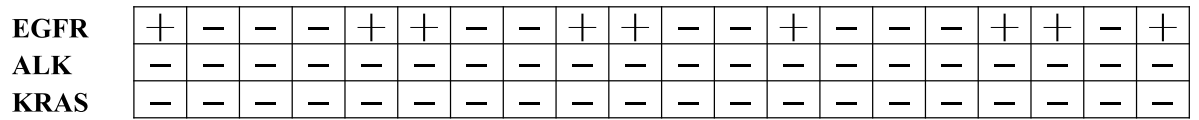

\begin{tabular}{|c|c|c|c|c|c|c|c|c|c|c|c|c|c|c|c|c|c|c|c|c|}
\hline Diagnosis & ¿ & 这 & 过 & $\underset{\mathscr{O}}{\mathscr{O}}$ & U & 导 & 导 & $\underset{\sim}{2}$ & 过 & U & 完 & U & 导 & $\underset{\mathscr{U}}{\tilde{O}}$ & $\underset{\mathscr{S}}{\circlearrowright}$ & $\underset{\mathscr{O}}{\mathrm{O}}$ & 巳 & 导 & 道 & 完 \\
\hline Smoking & $\mathrm{N}$ & $\mathrm{N}$ & $\mathrm{C}$ & $\mathrm{C}$ & $\mathrm{C}$ & $\mathrm{N}$ & $\mathrm{N}$ & E & $\mathrm{N}$ & $\mathrm{N}$ & $\mathrm{C}$ & $\mathrm{C}$ & $\mathrm{N}$ & $\mathrm{C}$ & E & $\mathrm{C}$ & $\mathrm{E}$ & $\mathrm{N}$ & $\mathrm{N}$ & $\mathrm{N}$ \\
\hline Гiming & M & M & M & M & $\mathrm{S}$ & $\mathrm{M}$ & $\mathrm{M}$ & $\mathrm{S}$ & M & M & $\mathrm{S}$ & $\mathrm{S}$ & $\mathrm{S}$ & $\mathrm{M}$ & $\mathrm{M}$ & $\mathrm{S}$ & M & M & $\mathrm{S}$ & M \\
\hline
\end{tabular}

Fig. 2 Immunohistochemical analysis of CD3, CD4, CD8, FOXP3, and PD-1 on tumor-infiltrating lymphocytes, and PD-L1 on tumor cells of patients in cohort 2. (Upper) Ladder plots demonstrate the different expression of each marker between primary lung cancer and brain metastases. Individual patients in cohort 2 are denoted as different colored lines. Statistical significance was estimated using a paired Wilcoxon rank-sum test. (Lower) Detailed information, including clinicodemographic characteristics, are summarized in this figure. Because lung biopsy tissues from 8 patients (patients $1,5,9,11,13,14,19,20$ ) were too small to be examined by $\mathrm{IHC}$, paired analysis was possible in only 12 out of 20 patients in cohort 2. Each colored tile in this figure represents the increased expression of each marker in the brain relative to that in primary lung cancers. Abbreviations: PD-1, programmed cell death-1; PD-L1, programmed cell death ligand-1; ID, identification number; EGFR, epidermal growth factor receptor; ALK, anaplastic lymphoma kinase; Erlo., erlotinib; Gefi., gefitinib; TKI, tyrosine kinase inhibitor; ADC, adenocarcinoma; SQC, squamous cell carcinoma; SAR, pulmonary sarcomatoid carcinoma; N, never smoked; C, current smoker; E, ex-smoker; M, metachronous; S, synchronous. a: Exon 19 deletion. b: L858R mutation

infiltration of $\mathrm{CD}^{+}$and $\mathrm{CD}^{+}$TILs was also decreased, especially for lung adenocarcinoma.

\section{Prognostic implication of immunological tumor microenvironment in metastatic brain tumors} To investigate the prognostic significance of immunological TME of brain metastasis, the OS rate of cohort 2 after brain surgery was studied in terms of the amount of $\mathrm{CD}^{+}$and $\mathrm{PD}^{+}$TILs, and PD-L1 expression on tumor cells. Since there were no clear-cut off points for these values, the median of each variable was arbitrarily used as a cut-off point. All patients in cohort 2 did not exhibit any survival differences (data not shown). For adenocarcinoma patients in cohort 2, however, a higher 

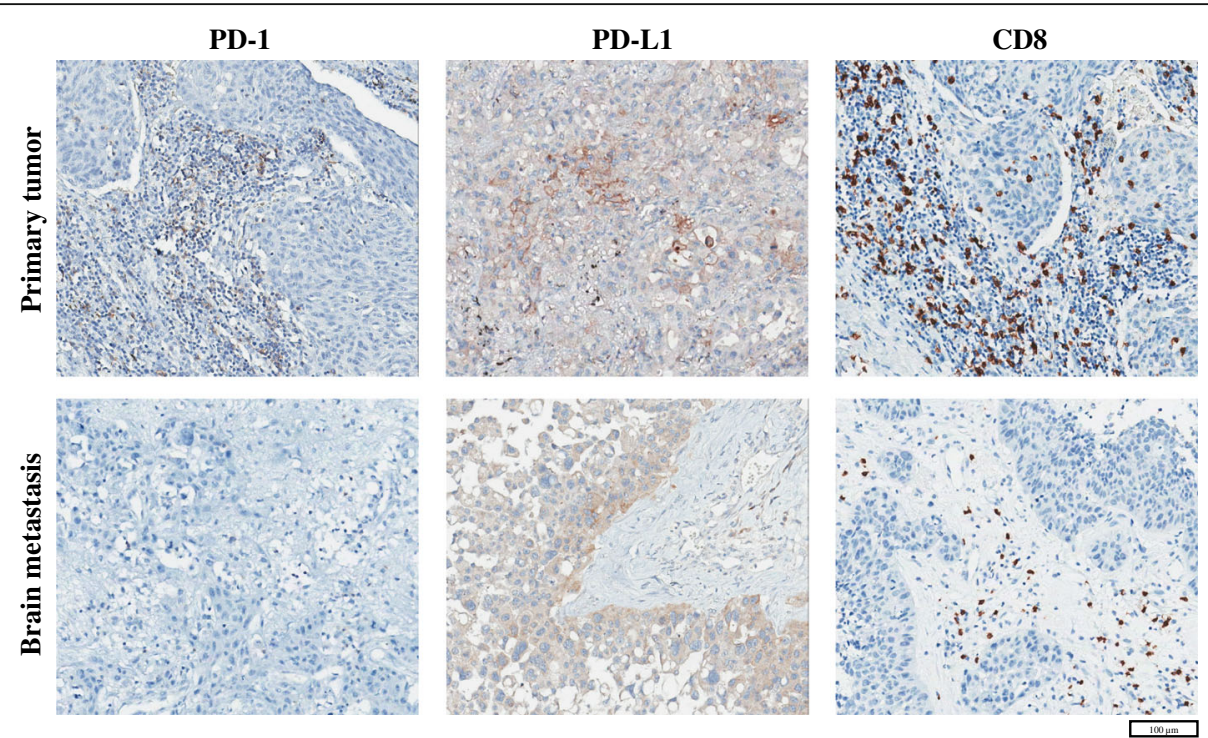

Fig. 3 Representative immunohistochemical images of PD-1, PD-L1, and CD8 in primary lung cancer and brain metastatic tissues of a patient from cohort 2 (patient ID 17). Abbreviations: PD-1, programmed cell death-1; PD-L1, programmed cell death ligand-1

infiltration of $\mathrm{CD}^{+}$TILs tended was associated with a worse prognosis (HR 2.47, 95\% CI 0.77-7.96; $P=0.130$, $\log$-rank $P=0.119$; Fig. 4a) Interestingly, patients showing a higher infiltration of $\mathrm{PD} 1^{+}$TILs exhibited a dismal prognosis that was statistically significant (HR, 1.20, 95\% CI, 1.01-11.04; $P=0.049$; log-rank $P=0.039$; Fig. 4b). In terms of PD-L1 expression on tumor cells, there was no prognostic implication according to the PD-L1 H-score (HR 1.24, 95\% CI 0.39-3.91; $P=0.711$, log-rank $P=$ 0.710; Fig. 4c). Collectively, this analysis showed that the immunological TME in the brain had a prognostic implication after brain metastasectomy, especially for patients with adenocarcinoma histology.

\section{Discussion}

In this study, we found that the BM of lung cancer did not respond well to ICIs. Immunohistochemical analysis revealed a decreased infiltration of $\mathrm{PD}-1^{+}$TILs in BM compared to primary lung cancer. These findings demonstrate that decreased PD $-1^{+}$of TILs could be one of the potential causative reasons for a poor intracranial response to ICIs.

Activated $\mathrm{T}$ cells have the potential to penetrate the blood-brain barrier (BBB) and readily infiltrate tumors within the brain, giving rise to the possibility that an ICI may have a therapeutic effect on BM [26]. However, the brain has been generally considered an immune privileged site that may dampen the therapeutic efficacy of ICIs because of the presence of BBB [27]. Although there has been no published pharmacokinetic or pharmacodynamics studies in on-treatment brain tissue to allow determination of drug penetration into the tumor, some preclinical data demonstrated ICI's penetration to $\mathrm{BBB}$ for primary brain tumors and for metastatic tumors. [28] Furthermore, for melanoma patients with stable asymptomatic brain metastases, ipilimumab, and to a lesser extent pembrolizumab, did show any activity, with half of all patients achieving a partial response or stable disease [29]. Whether this also applies to lung cancer patients is currently being investigated in a number of early phase immunotherapy trials.

An recent interim analysis of a phase II study showed that pembrolizumab may have a role in NSCLC patients with brain metastases [19]. Unfortunately, however, many patients did not respond to pembrolizumab in the brain or in extracranial sites. Similarly, only $16.7 \%$ of the patients in cohort 1 of the present study responded to treatment for BM with anti-PD-1 antibody. Although two patients achieved a partial response, they underwent WBRT with anti-PD-1 antibody, making it difficult to interpret the ICI response. The superior response rate observed in the phase II study might be due to exclusion of patients with any history of BM or active/symptomatic BM. In general, there is huge gap between clinical trials and real world lung cancer patients because of strict criteria for patient enrollment of clinical trials. [30] Our study represents that the BM of lung cancer patient in real world do not seem to respond well to ICIs.

Over the past decades, the TME has been considered a fundamental regulator of cancer progression and therapeutic efficacy. The TME contains various noncancerous cell types, including endothelial cells, pericytes, fibroblasts, and immune cells [21]. While several of these cell types are common to both, there are significant differences between the TMEs of primary lung cancer and the 


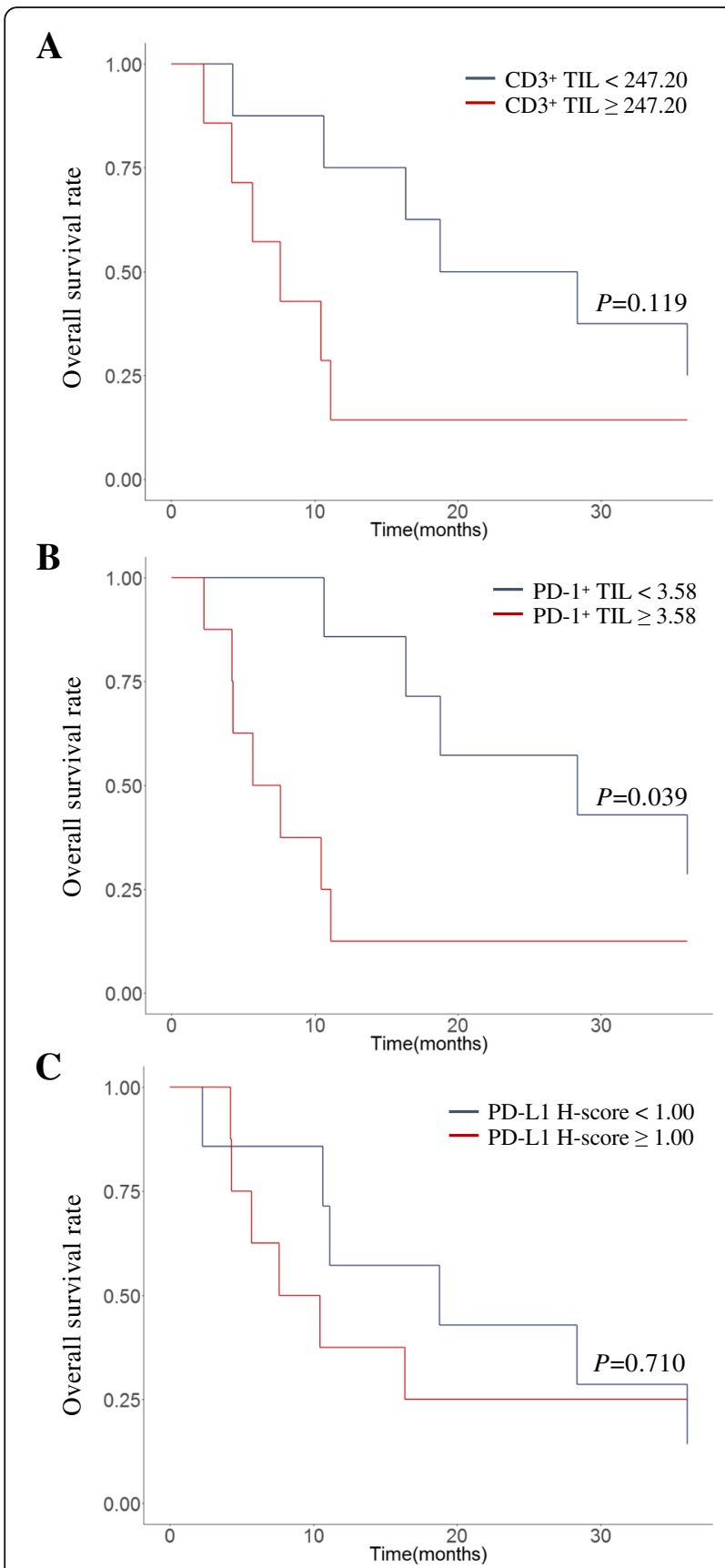

Fig. 4 Kaplan-Meier plots for lung cancer patients with adenocarcinoma histology in cohort 2 according to $\mathbf{a}$ the amount of $\mathrm{CD}^{+}{ }^{+} \mathrm{TILS}$, b PD1 ${ }^{+}$TILs, and $\mathbf{c}$ the PD-L1 H-score in brain metastatic tissue. Overall survival was measured from the date of surgery until either death due to any cause or the last follow-up date. The cutoffpoints were arbitrarily set to the median value of each variable. The significance of differences in survival curves were compared using a log-rank test. Abbreviations: TIL, tumor-infiltrating lymphocyte; PD-1, programmed cell death-1; PD-L1, programmed cell death ligand-1 brain, allowing mechanisms that regulate tumor cell niches in the brain to differ from those of other tissues $[22,23]$. In order to explain why BM were not responsive to ICIs, attention was paid to the immunological environment in the TME of the brain. The brain TME was found to have lost PD-1 $1^{+}$TILs despite their presence in primary lung cancer specimens, which is a novel finding of this study. In accordance with others [31-33], adenocarcinoma patients displayed a more distinctive pattern of a low infiltration of both $\mathrm{CD}^{+}$and $\mathrm{CD} 8^{+}$TILs, suggesting that a significant number of patients lack any significant local immune response in association with their BM. Although PD-L1 expression of tumor cells showed no significant differences in this study, others have reported that BM lost PD-L1 expression that was normally present in primary lung cancer specimens [22, 34]. Overall, such an immunological heterogeneity may explain, at least in part, the different therapeutic responses to PD-1 inhibitors.

While limited data suggest that the intracranial response to ICIs may have the distinct advantage of producing durable responses in selected patients [19], there is no definitive biomarker to identify this population. $\mathrm{Al}$ though there have been significant efforts to develop PD-L1 as a predictive biomarker to select patients for treatment with PD-1 or PD-L1 inhibitors, consensus criteria for evaluating PD-L1 as a predictive biomarker have not yet been settled [35]. As indicated in our study, the immunological TME in the brain, including the infiltration of PD- $1^{+}$TILs, may be important in predicting the clinical benefits of PD-1/PD-L1 checkpoint blockades in lung cancer patients with BM. Therefore, we suggest that the role of PD-1 as a biomarker in the brain TME should be investigated in future clinical studies of immunotherapy in lung cancer with BM. When physicians decide to treat patients with a PD-1 or PD-L1 inhibitor, the distinctive immunological environment of the brain should be taken into consideration.

This study has several limitations. The two cohorts analyzed in this study were small due to the limited number of patients with BM who underwent anti-PD-1 antibody treatment or a brain metastasectomy. Furthermore, since the two cohorts were independent of each other, the predictive role of PD-1 for anti-PD-1 antibody treatment could not be evaluated directly. However, it was sufficient to generate the hypothesis that PD-1 could be a potential biomarker. Actually, some of the progressive diseases observed in cohort 1 could have been pseudoprogressions. However, the main purpose of this study was to investigate the difference in treatment response between BM and primary lung cancer, rather than the absolute response of each organ. Therefore, the existence of pseudoprogression is unlikely to change the conclusion of this study. 


\section{Conclusions}

Our findings suggest that there may be discordant extra/ intracranial responses to ICIs. The diminished infiltration of PD- $1^{+}$TILs into tumor tissue in the brain may be one of potential factors that hinders the response to anti-PD-1 antibody in the BM. These findings provide a rationale for studying the infiltration of PD- $1^{+}$TILs in the brain as a potential biomarker for future immunotherapy trials that incorporate patients with BM. Hopefully, ongoing studies will improve treatment selection strategies for lung cancer patients with BM.

\section{Additional files}

Additional file 1: Figure S1. Representative images for response evaluation in brain metastasis. An intracranial response was assessed by brain gadolinium-enhanced magnetic resonance imaging, using Response Evaluation Criteria in Solid Tumors modified to allow target central nerve system lesions, $5 \mathrm{~mm}$ or larger in maximum diameter, and with up to five BMs permitted (modified RECIST). Each patient received either nivolumab $(N)$ or pembrolizumab $(P)$ for the specified number of cycles. Abbreviations: ADC, adenocarcinoma; $\mathrm{SCL}$, smallcell lung cancer; SAR, pulmonary sarcomatoid carcinoma; $P$, pembrolizumab; N, nivolumab; PD, progressive disease; $P R$, partial response; SD, stable disease. (TIF $2970 \mathrm{~kb}$ )

Additional file 2: Figure S2. Immunohistochemical analysis of CD3, CD4, CD8, FOXP3, and PD-1 on tumor-infiltrating lymphocytes, and PD-L1 on tumor cells of patients with an adenocarcinoma histology in cohort 2. Ladder plots demonstrate the different expression of each marker between primary lung cancer and brain metastases. Individual patients are denoted as different colored lines. Statistical significance was estimated using a paired Wilcoxon rank sum test. (TIF 497 kb)

Additional file 3: Figure S3. Scatter plots demonstrating the correlation between PD-L1 expression on tumor cells and the amount of $\mathrm{CD}^{+}{ }^{+} \mathrm{TILs}$ or $\mathrm{PD} 1^{+}$TILs in primary lung cancer specimens, and metastatic brain tumors. Correlation was evaluated by Spearman's rank correlation analysis. Abbreviations: PD-L1, programmed cell death ligand-1; TIL, tumor-infiltrating lymphocyte. (TIF 379 kb)

Additional file 4: Figure S4. Scatter plot demonstrating the correlation between infiltrating PD- $1^{+}$tumor-infiltrating lymphocyte on brain metastasis and the interval of development of brain metastasis. Correlation was evaluated by Spearman's rank correlation analysis. Abbreviations: PD-1, programmed cell death-1; TIL, tumor-infiltrating lymphocyte. (TIF 143 kb)

\section{Abbreviations}

ADC: Adenocarcinoma; ALK: Anaplastic lymphoma kinase; EGFR: Epidermal growth factor receptor; ID: Identification number; N: Nivolumab; P: Pembrolizumab; PD: Progressive disease; PD-1: Programmed cell death-1; PD-L1: Programmed cell death ligand-1; PR: Partial response; SAR: Pulmonary sarcomatoid carcinoma; SCL: Small-cell lung cancer; SD: Stable disease; SQC: Squamous cell carcinoma; TIL: Tumor-infiltrating lymphocyte

\section{Acknowledgements}

NSCLC Expanded Access Program for nivolumab was sponsored by ONO PHARMA KOREA CO., LTD. We would like to thank BioMed Proofreading LLC for English editing.

\section{Funding}

This study was supported by grants from the Seoul National University Hospital Research Fund [grant number 03-2015-0380]. The funding bodies had no role in the design of the study and collection, analysis, and interpretation of data and in writing the manuscript.

\section{Availability of data and materials}

The datasets used and/or analysed during the current study are available from the corresponding author on reasonable request.

\section{Authors' contributions}

Designing the concept of the study: RK, BK. Provision of study patients and chemotherapy: BK, MK, SHK, YJK, TMK, DK, JSL, DSH. Provision of study patients and surgery: JWK. Pathologic analysis: SK, YK, DHC. Data gathering, statistical analysis and interpretation: RK, BK. Manuscript writing: RK. All authors read and approved the final manuscript.

\section{Ethics approval and consent to participate}

This study was approved by the SNUH Institutional Review Board (IRB approval number: $\mathrm{H}-1702-158-836)$ and was conducted in accordance with Declaration of Helsinki provisions. Written informed consent to participate in the study was obtained from each patient at admission.

\section{Consent for publication}

Not applicable.

\section{Competing interests}

The authors declare that they have no competing interests.

\section{Publisher's Note}

Springer Nature remains neutral with regard to jurisdictional claims in published maps and institutional affiliations.

\section{Author details}

${ }^{1}$ Department of Internal Medicine, Seoul National University Hospital, 101 Daehak-ro, Jongno-gu, Seoul 03080, Republic of Korea. ${ }^{2}$ Cancer Research Institute, Seoul National University College of Medicine, 101 Daehak-ro, Jongno-gu, Seoul 03080, South Korea. ${ }^{3}$ Department of Pathology, Seoul National University Hospital, 101 Daehak-ro, Jongno-gu, Seoul 03080, South Korea. ${ }^{4}$ Department of Internal Medicine, Seoul National University Bundang Hospital, 82 Gumi-ro 173 Beon-gil, Bundang-gu, Seongnam-si, Gyeonggi-do 13620, South Korea. ${ }^{5}$ Department of Neurosurgery, Seoul National University Hospital, 101 Daehak-ro, Jongno-gu, Seoul 03080, South Korea.

Received: 23 November 2017 Accepted: 12 December 2018 Published online: 07 January 2019

\section{References}

1. Nayak L, Lee EQ, Wen PY. Epidemiology of brain metastases. Curr Oncol Rep. 2012;14(1):48-54.

2. Sorensen JB, Hansen $\mathrm{HH}$, Hansen M, Dombernowsky P. Brain metastases in adenocarcinoma of the lung: frequency, risk groups, and prognosis. Journal of clinical oncology : official journal of the American Society of Clinical Oncology. 1988;6(9):1474-80

3. Mathieu D, Kondziolka D, Cooper PB, Flickinger JC, Niranjan A, Agarwala S, Kirkwood J, Lunsford LD. Gamma knife radiosurgery for malignant melanoma brain metastases. Clin Neurosurg. 2007;54:241-7.

4. Alomari A, Rauch PJ, Orsaria M, Minja FJ, Chiang VL, Vortmeyer AO. Radiologic and histologic consequences of radiosurgery for brain tumors. J Neuro-Oncol. 2014;117(1):33-42.

5. Greene-Schloesser D, Robbins ME: Radiation-induced cognitive impairmentfrom bench to bedside. Neuro-oncology 2012, 14 Suppl 4:iv37-iv44.

6. Khan AJ, Dicker AP. On the merits and limitations of whole-brain radiation therapy. Journal of clinical oncology : official journal of the American Society of Clinical Oncology. 2013;31(1):11-3.

7. Lee DH, Han JY, Kim HT, Yoon SJ, Pyo HR, Cho KH, Shin SH, Yoo H, Lee SH, Lee JS. Primary chemotherapy for newly diagnosed nonsmall cell lung cancer patients with synchronous brain metastases compared with wholebrain radiotherapy administered first : result of a randomized pilot study. Cancer. 2008;113(1):143-9.

8. Attia A, Page BR, Lesser GJ, Chan M. Treatment of radiation-induced cognitive decline. Curr Treat Options in Oncol. 2014;15(4):539-50.

9. Peters S, Adjei AA, Gridelli C, Reck M, Kerr K, Felip E: Metastatic non-smallcell lung cancer (NSCLC): ESMO Clinical Practice Guidelines for diagnosis, treatment and follow-up. Annals of oncology : official journal of the European Society for Medical Oncology 2012, 23 Suppl 7:vii56-vii64. 
10. Dong H, Strome SE, Salomao DR, Tamura H, Hirano F, Flies DB, Roche PC, Lu J, Zhu G, Tamada K, et al. Tumor-associated B7-H1 promotes T-cell apoptosis: a potential mechanism of immune evasion. Nat Med. 2002;8(8): 793-800.

11. Freeman GJ, Long AJ, Iwai Y, Bourque K, Chernova T, Nishimura H, Fitz LJ, Malenkovich N, Okazaki T, Byrne MC, et al. Engagement of the PD-1 immunoinhibitory receptor by a novel B7 family member leads to negative regulation of lymphocyte activation. J Exp Med. 2000;192(7):1027-34.

12. Carbone DP, Reck M, Paz-Ares L, Creelan B, Horn L, Steins M, Felip E, van den Heuvel MM, Ciuleanu TE, Badin F, et al. First-line Nivolumab in stage IV or recurrent non-small-cell lung Cancer. N Engl J Med. 2017;376(25):2415-26.

13. Borghaei H, Paz-Ares L, Horn L, Spigel DR, Steins M, Ready NE, Chow LQ, Vokes EE, Felip E, Holgado E, et al. Nivolumab versus docetaxel in advanced nonsquamous non-small-cell lung Cancer. N Engl J Med. 2015;373(17):1627-39.

14. Brahmer J, Reckamp KL, Baas P, Crino L, Eberhardt WE, Poddubskaya E, Antonia S, Pluzanski A, Vokes EE, Holgado E, et al. Nivolumab versus docetaxel in advanced squamous-cell non-small-cell lung Cancer. N Engl J Med. 2015;373(2):123-35.

15. Garon EB, Rizvi NA, Hui R, Leighl N, Balmanoukian AS, Eder JP, Patnaik A, Aggarwal C, Gubens M, Horn L, et al. Pembrolizumab for the treatment of non-small-cell lung cancer. N Engl J Med. 2015;372(21):2018-28.

16. Robert C, Long GV, Brady B, Dutriaux C, Maio M, Mortier L, Hassel JC, Rutkowski P, McNeil C, Kalinka-Warzocha E, et al. Nivolumab in previously untreated melanoma without BRAF mutation. N Engl J Med. 2015;372(4):320-30

17. Robert C, Schachter J, Long GV, Arance A, Grob JJ, Mortier L, Daud A, Carlino MS, McNeil C, Lotem M, et al. Pembrolizumab versus Ipilimumab in Advanced Melanoma. N Engl J Med. 2015;372(26):2521-32.

18. Tan AC, Heimberger AB, Menzies AM, Pavlakis N, Khasraw M. Immune checkpoint inhibitors for brain metastases. Curr Oncol Rep. 2017;19(6):38.

19. Goldberg SB, Gettinger SN, Mahajan A, Chiang AC, Herbst RS, Sznol M, Tsiouris AJ, Cohen J, Vortmeyer A, Jilaveanu L, et al. Pembrolizumab for patients with melanoma or non-small-cell lung cancer and untreated brain metastases: early analysis of a non-randomised, open-label, phase 2 trial. The Lancet Oncology. 2016;17(7):976-83.

20. Margolin K, Ernstoff MS, Hamid O, Lawrence D, McDermott D, Puzanov I, Wolchok JD, Clark JI, Sznol M, Logan TF, et al. Ipilimumab in patients with melanoma and brain metastases: an open-label, phase 2 trial. The Lancet Oncology. 2012;13(5):459-65.

21. Quail DF, Joyce JA. Microenvironmental regulation of tumor progression and metastasis. Nat Med. 2013;19(11):1423-37.

22. Mansfield AS, Aubry MC, Moser JC, Harrington SM, Dronca RS, Park SS, Dong $\mathrm{H}$. Temporal and spatial discordance of programmed cell deathligand 1 expression and lymphocyte tumor infiltration between paired primary lesions and brain metastases in lung cancer. Annals of oncology : official journal of the European Society for Medical Oncology. 2016;27(10): 1953-8.

23. Quail DF, Joyce JA. The microenvironmental landscape of brain tumors. Cancer Cell. 2017;31(3):326-41.

24. Qian JM, Mahajan A, Yu JB, Tsiouris AJ, Goldberg SB, Kluger HM, Chiang VLS. Comparing available criteria for measuring brain metastasis response to immunotherapy. J Neuro-Oncol. 2017:1-7.

25. Koh J, Go H, Keam B, Kim MY, Nam SJ, Kim TM, Lee SH, Min HS, Kim YT, Kim DW, et al. Clinicopathologic analysis of programmed cell death-1 and programmed cell death-ligand 1 and 2 expressions in pulmonary adenocarcinoma: comparison with histology and driver oncogenic alteration status. Modern pathology : an official journal of the United States and Canadian Academy of Pathology, Inc. 2015;28(9):1154-66.

26. Prins RM, Vo DD, Khan-Farooqi H, Yang MY, Soto H, Economou JS, Liau LM, Ribas A. NK and CD4 cells collaborate to protect against melanoma tumor formation in the brain. Journal of immunology. 2006;177(12):8448-55.

27. Carson MJ, Doose JM, Melchior B, Schmid CD, Ploix CC. CNS immune privilege: hiding in plain sight. Immunol Rev. 2006;213:48-65.

28. Cohen JV, Kluger HM. Systemic immunotherapy for the treatment of brain metastases. Front Oncol. 2016;6:49.

29. Schadendorf D, Hodi FS, Robert C, Weber JS, Margolin K, Hamid O, Patt D, Chen TT, Berman DM, Wolchok JD. Pooled analysis of Long-term survival data from phase II and phase III trials of Ipilimumab in Unresectable or metastatic melanoma. Journal of clinical oncology : official journal of the American Society of Clinical Oncology. 2015;33(17):1889-94.
30. Yoo MK SH, Keam B, Kim TM, Kim D, Heo DS. Generalization and representativeness of phase III immune checkpoint inhibitor trials in NSCLC. Ann Oncol. 2017;28:736-44.

31. Berghoff AS, Inan C, Ricken G, Widhalm G, Dieckmann K, Birner P, Oberndorfer F, Dome B, Bartsch R, Zielinski C et al: 1324PTUMORINFILTRATING LYMPHOCYTES (TILS) AND PD-L1 EXPRESSION IN NONSMALL CELL LUNG CANCER BRAIN METASTASES (BM) AND MATCHED PRIMARY TUMORS (PT). Annals of Oncology 2014, 25(suppl_4):iv465-iv466.

32. Harter PN, Bernatz S, Scholz A, Zeiner PS, Zinke J, Kiyose M, Blasel S, Beschorner R, Senft C, Bender B, et al. Distribution and prognostic relevance of tumor-infiltrating lymphocytes (TILs) and PD-1/PD-L1 immune checkpoints in human brain metastases. Oncotarget. 2015;6(38):40836-49.

33. Teglasi V, Reiniger L, Fabian K, Pipek O, Csala I, Bago AG, Varallyai P, Vizkeleti $L$, Rojko L, Timar J, et al. Evaluating the significance of density, localization, and PD-1/PD-L1 immunopositivity of mononuclear cells in the clinical course of lung adenocarcinoma patients with brain metastasis. NeuroOncology. 2017;19(8):1058-67.

34. Kluger HM, Zito CR, Barr ML, Baine MK, Chiang VL, Sznol M, Rimm DL, Chen L, Jilaveanu LB. Characterization of PD-L1 expression and associated T-cell infiltrates in metastatic melanoma samples from variable anatomic sites. Clinical cancer research : an official journal of the American Association for Cancer Research. 2015;21(13):3052-60.

35. Kerr KM, Nicolson MC. Non-small cell lung Cancer, PD-L1, and the pathologist. Arch Pathol Lab Med. 2016;140(3):249-54.

\section{Ready to submit your research? Choose BMC and benefit from:}

- fast, convenient online submission

- thorough peer review by experienced researchers in your field

- rapid publication on acceptance

- support for research data, including large and complex data types

- gold Open Access which fosters wider collaboration and increased citations

- maximum visibility for your research: over $100 \mathrm{M}$ website views per year

At BMC, research is always in progress.

Learn more biomedcentral.com/submissions 